\title{
Estimativa de custos diretos para a implantação e efetivação da vacinação contra o HPV no Estado do Amazonas
}

\section{Estimation of direct costs for the implantation and effectiveness of vaccination against HPV in the State of Amazonas}

\author{
Patrícia da Silva Magalhães ${ }^{1}$, André Luiz Machado das Neves ${ }^{2}, Z_{1}$ ilmar Augusto de Souza Filho ${ }^{3}$
}

\begin{abstract}
Resumo
O estudo estimou os custos diretos da implantação e efetivação das campanhas de vacinação contra o HPV no Estado do Amazonas. Trata-se de um estudo transversal prospectivo, com uso de modelos matemáticos e descritivo de avaliação econômica em saúde. Utilizou-se dados primários e secundários para estimativa dos custos envolvidos nas campanhas realizadas entre os anos de 2013 a 2014. Avaliou-se os custos através da análise de correlação de Pearson, com valores significativos de $p \leq 0,05$. Identificou-se que no Amazonas, o percentual de cobertura da campanha para a $1^{\text {a }}$ dose foi de $95,4 \%$, na $2^{\mathrm{a}}$ dose a cobertura foi de $94,4 \%$ e na $3^{\mathrm{a}}$ dose $84,1 \%$ da população de adolescentes do sexo feminino. Os custos diretos totais estimados para as três etapas da campanha, revelaram o valor de R $\$ 16.149 .814,79$, aplicados através da Secretaria de Estado de Saúde do Amazonas, bem como os custos apresentaram correlação estatisticamente significativa com a variável população alvo $(\mathrm{p}<0,001)$. A realização de campanhas de vacinação no Amazonas é muito onerosa e deve-se considerar a complexidade e dinamicidade da territorialidade do Estado e os recursos humanos, fazendo-se necessário a aplicação do princípio da equidade no processo de planejamento das ações de saúde para a implantação e efetivação de campanhas de vacinação na Amazônia.
\end{abstract}

Palavras chave: Gastos em saúde. Custos e análise de custo. Vacina. HPV.

\begin{abstract}
The study estimated the direct costs of implementing and conducting vaccination campaigns against HPV in the state of Amazonas. This is a cross-sectional prospective study, using mathematical models and descriptive of economic evaluation in health. Primary and secondary data were used to estimate the costs involved in the campaigns carried out between the years of 2013 and 2014. The costs were evaluated through the Pearson correlation analysis, with significant values of $p \leq 0.05$. It was identified that in the Amazon, the percentage coverage of the campaign for the 1 st dose was $95.4 \%$, in the second dose to the coverage was $94.4 \%$ and in the third dose $84.1 \%$ of the female adolescents. The total direct costs estimated for the three stages of the campaign, revealed the value of $\mathrm{R} \$ 16,149,814.79$, applied through the State Department of Health of Amazonas, as well as costs presented a statistically significant correlation with the target population variable $(\mathrm{p}<0.001)$. The vaccination campaigns in Amazonas are very expensive and the complexity and dynamicity of the state's territoriality and human resources must be considered, making it necessary to apply the principle of equity in the process of planning health actions for the implantation and the implementation of vaccination campaigns in the Amazon.
\end{abstract}

Keywords: Health expenditures. Costs and cost analysis. Vaccine. HPV.

\footnotetext{
${ }^{1}$ Mestre em Saúde Coletiva pelo Instituto Sírio-Libanês de Ensino e Pesquisa, São Paulo, São Paulo, Brasil. Enfermeira da Secretaria Estadual de Saúde do Amazonas, Manaus, Amazonas, Brasil.

${ }^{2}$ Doutorando em Saúde Coletiva pela Universidade do Estado do Rio de Janeiro, Rio de Janeiro, Brasil. Professor da Escola Superior de Ciências da Saúde da Universidade do Estado do Amazonas, Manaus, Amazonas, Brasil. E-mail: andre machadostm@hotmail.com

${ }^{3}$ Doutor em Enfermagem na Saúde do Adulto pela Universidade de São Paulo, São Paulo, Brasil. Professor da Escola de Enfermagem de Manaus da Universidade Federal do Amazonas, Manaus, Amazonas, Brasil.
} 


\section{Introdução}

O câncer do colo do útero, também chamado de cervical, demora entre 10 a 20 anos para se desenvolver. ${ }^{(1)}$ Durante algum tempo foi considerado como uma doença dos países desenvolvidos e com grandes recursos financeiros. ${ }^{(2)}$ Atualmente, em países em desenvolvimento, como o Brasil, por exemplo, ele é o segundo tumor mais frequente na população feminina, atrás apenas do câncer de mama. ${ }^{(2-3)}$ Segundo a Diretoria de Vigilância Epidemiológica - DIVE ${ }^{(4)}, \mathrm{o}$ câncer de colo do útero manifesta-se a partir da faixa etária de 25 a 29 anos, aumentando seu risco até a faixa etária de 50 a 60 anos. A estimativa (para o biênio 2016-2017) de incidência do câncer do colo do útero no Brasil, conforme o Instituto Nacional de Câncer José Alencar Gomes da Silva - INCA ${ }^{(5)}$, apresenta um risco estimado de 15,85 casos a cada 100 mil mulheres.

O câncer do colo do útero é o mais incidente na região Norte $(23,57 / 100$ mil) sendo que no estado do Amazonas a estimativa para 2014 foi de 33,51/100 mil, superando o câncer de mama (21,75/100 mil), como o câncer que fica em primeiro lugar no ranking de incidência entre a população do Estado. ${ }^{(6)}$

A principal alteração que pode levar ao câncer de colo uterino é a infecção pelo HPV. Assim sendo, a vacina contra o HPV é uma ferramenta substancial para o combate desse câncer. Porém, ainda é uma prática distante da realidade dos países em desenvolvimento e subdesenvolvidos, em razão de seu alto custo. No ano de 2014, o Brasil foi o oitavo país da América do Sul a disponibilizar a vacina contra HPV de modo gratuito para a população. Neste aspecto, ao referirem-se sobre o crescimento na incidência de câncer, destacam os gastos para seu controle como um problema de saúde pública. ${ }^{(7)}$

Em 1999, o Ministério da Saúde despendeu R\$ 470 milhões para o tratamento de 156 mil pacientes portadores de câncer. Nove anos após, em 2008, houve um incremento de $176,4 \%$ desses custos, ultrapassando R $\$ 1,7$ bilhões, apesar de um aumento proporcionalmente menor $(60,9 \%)$ no número de pacientes tratados pelo Sistema Único de Saúde (SUS).

A vacinação, em conjunto com as atuais ações para o rastreamento do câncer no colo do útero, visa possibilitar nas próximas décadas a prevenção dessa doença, que representa hoje uma das principais causas de morbidade por neoplasias entre mulheres no Brasil.(4). O Ministério da Saúde, por meio do Programa Nacional de Imunizações (PNI), ampliou o
Calendário Nacional de Vacinação com a inserção da vacina quadrivalente contra o papilomavírus humano (HPV) no Sistema Único de Saúde (SUS) no ano de 2014. ${ }^{(4)}$ Ressalta-se que o Amazonas, no ano de 2013, foi o primeiro na região norte do Brasil a fornecer gratuitamente a vacina contra o HPV, antes de ser disponibilizada no calendário nacional de vacinação.

Uma das questões relevantes em políticas públicas de saúde é mensurar a eficiência econômica das mesmas em termos de custos e benefícios. ${ }^{(8-10)}$ Entender que para adoção de nova tecnologia, mesmo que em âmbito estadual, é necessário um estudo da efetividade da adoção de novas medidas na área de saúde, comparando o custo com os efeitos benéficos para a sociedade.

No estado do Amazonas, mensurar o quanto uma política de saúde pode comprometer o orçamento da saúde tornou-se ainda mais relevante, dado os problemas orçamentários em Estados mais pobres da União.

Diante dessas demandas, o objetivo deste artigo é estimar os custos diretos da implantação e efetivação da campanha de vacinação contra o HPV no Estado do Amazonas, há ainda uma lacuna de estudos sobre implantação de novas vacinas em programas de imunização nacional, situação que se torna mais difícil quando se trata de programas escolares, principalmente, em países em desenvolvimento. Por isso a necessidade desse estudo, porque ele estuda a estimativa de custos para a efetivação da campanha de vacinação contra o HPV no estado do Amazonas, em adolescentes escolares. $^{(11-12)}$

\section{Material e Método}

Trata-se de um estudo transversal prospectivo de avaliação econômica em saúde do tipo de modelos matemáticos seguindo a perspectiva de Vanni et al. ${ }^{(13)}$, e descritivo de acordo com a conceituação de Polit e Becker. ${ }^{(14)}$

Sob o tipo de avaliação econômica em saúde seguindo os modelos matemáticos, Vanni et al. ${ }^{(13)}$, consideram que o desenvolvimento de modelos matemáticos que utilizam dados da literatura e dos bancos de dados disponíveis é o método mais comumente utilizado para a realização de estudos econômicos, por permitirem integrar dados de diferentes fontes e comparar diferentes cenários. Os modelos matemáticos devem representar os eventos relevantes da história natural de uma doença, bem 
como os efeitos das intervenções. Avaliação econômica em saúde compreende a comparação de alternativas com respeito a seus custos e consequências. Portanto, a tarefa básica de qualquer avaliação econômica é identificar, medir, valorar e comparar os custos e consequências das alternativas.

No que tange ao aspecto descritivo, o estudo delimitou-se na exploração da compreensão do perfil da distribuição, dos custos da campanha de vacinação contra o HPV com a finalidade de observar sua natureza complexa. Nesta acepção, tem-se o objetivo central de retratar de modo correto as características das pessoas, situações ou grupos e/ou a frequência que os fenômenos ocorrem..$^{(14)}$

\section{Caracterização de custos diretos}

A despeito dos custos diretos, foram definidas as seguintes variáveis: beneficiárias, valor da dose para o estado, contrapartida estadual e logística de entrega das vacinas. Baseando-se na concepção dos estudos de Wichmann e Silva ${ }^{(15)}$ descrevem-se as definições supracitadas para os custos diretos:

- Beneficiárias: mulheres nascidas entre os anos de 2000 a 2002, no ato de vacinar contra o HPV no Estado do Amazonas conforme as escolhas operacionais da Secretaria de Estado de Saúde do Amazonas (SUSAM).

- Valor por dose: faz referência ao custo de comercialização da vacina contra o HPV. Neste caso, o preço da comercialização da dose da vacina escolhida pela SUSAM foi $\mathrm{R} \$ 42,00$. Essa vacina era do tipo Bivalente (tipos 16 e 18) registrado com a marca de Cervarix ${ }^{\circledR}$, do laboratório GlaxoSmithKline.

- Logística da entrega: refere-se ao custo da entrega das vacinas e compreende a saída da capital (Manaus) até aos destinos finais (61 Municípios). Os custos variaram em função da distância (22 km o mais próximo e 1628 $\mathrm{km}$ o mais distante), tipo de transporte (fluvial e/ou aérea e/ou terrestre).

- Contrapartida estadual: refere-se aos valores (monetários) disponibilizados pela SUSAM para despesas locais (gelo, alimentos, combustíveis, material de divulgação, caixa térmica, óleo lubrificante, entre outros).

\section{Procedimentos de coleta de dados}

Neste estudo, foram utilizados dados secundários para estimativa dos custos envolvidos na campanha de vacinação. Solicitou-se a anuência da Secretaria Executiva Adjunta do Interior (SEAASI) da SUSAM. Os registros existentes são provenientes da Fundação de Vigilância em Saúde (FVS/SUSAM), referente à campanha de vacinação de acordo com o esquema vacinal cuja primeira dose iniciou no mês de julho/2013; a segunda no mês de setembro/2013 e terceira no mês de março/2014. Desta forma, destacase que este estudo utilizou fonte de dados secundários e não envolveu sujeitos humanos, não sendo necessário a obtenção de uma aprovação do Comitê de Ética e Pesquisa.

Elaborou-se um questionário, no formato de planilha digital através do software da Microsoft Office365, Excel versão 2013, enviados via e-mail para as Secretarias de Saúde de 61 municípios do Estado do Amazonas, para que seus respectivos secretários de saúde pudessem fornecer as seguintes informações: (a) público-alvo; (b) população imunizada; (c) cobertura; (d) valor da dose; (e) total da logística; (f) contrapartida do estado do Amazonas; (g) custo total e (h) valor unitário, de acordo com a realidade municipal.

Obteve-se o total de 20 questionários respondidos dos seguintes municípios: Apuí; Atalaia do Norte; Barcelos; Beruri; Boa Vista do Ramos; Boca do Acre; Borba; Caapiranga; Carauari; Eirunepé; Ipixuna; Itacoatiara; Itamarati; Itapiranga; Maués; Nhamundá; Novo Airão, Santa Isabel do Rio Negro; São Sebastião do Uatumã e Uarini. Os dados da campanha para a aplicação da primeira dose da vacina do HPV foram referentes ao mês de setembro de 2013; os da segunda dose do mês de dezembro de 2013 e o da terceira e última dose de julho de 2014.

\section{Organização e análise dos dados}

Para organização dos dados da população beneficiada e os dados dos custos diretos, utilizouse a planilha do software da Microsoft Office 365 Excel versão 2013, para facilitar a organização dos dados e análises matemáticas dos dados. O grau de correlação entre as variáveis foi avaliado por meio do coeficiente de correlação de Pearson, testando-se a significância estatística. Elegeu-se como variável dependente: os custos; e as variáveis independentes foram representadas pelos seguintes indicadores: 
a) Distância em linha reta $(\mathrm{km})$ da capital, b) Área territorial $\left(\mathrm{km}^{2}\right)$ e c) População alvo. As análises foram realizadas utilizando-se o software SPSS for Windows (versão 21.0). Considerou-se o nível de significância de $5 \%$ em todos os testes realizados.

\section{Resultados}

\section{Levantamento populacional das adolescentes beneficiadas pela campanha de vacinação}

Para a efetivação da campanha de vacinação contra o HPV no Amazonas, participaram adolescentes da capital e dos 61 municípios do interior do estado. A descrição estratificada por municípios com dados do ano de 2013-2014 cedidos pela Diretoria TécnicoCientífica (DITEC) da FVS do estado.

Tabela 1 - Descrição da População e Municípios atendidos na campanha de vacinação contra o HPV

\begin{tabular}{ccccc}
$\begin{array}{c}\text { Número de } \\
\text { municípios } \\
\text { TOTAL }\end{array}$ & $\begin{array}{c}\text { Tamanho da } \\
\text { população } \\
\text { alvo }\end{array}$ & $\begin{array}{c}\text { Cobertura } \\
\text { da } \mathbf{1}^{\mathbf{a}} \text { dose }\end{array}$ & $\begin{array}{c}\text { Cobertura } \\
\text { da 2 } 2^{\mathbf{a}} \text { dose }\end{array}$ & $\begin{array}{c}\text { Cobertura } \\
\text { da 3 } 3^{\mathbf{a}} \text { dose }\end{array}$ \\
\hline 62 & 108.283 & 103.350 & 97.584 & $82.065^{4}$ \\
\hline
\end{tabular}

Os dados evidenciaram que as adolescentes públicoalvo da campanha se encontravam na faixa etária entre 11 a 13 anos. Utilizou o Censo Escolar, do ano de 2012, que totalizou 108.283 adolescentes consideradas aptas à vacinação no Estado do Amazonas.

A partir desse contexto, na campanha de vacinação para aplicação da $1^{\text {a }}$ dose, constatou-se que no Amazonas, 103.350 das meninas foram vacinadas, com alto grau de cobertura, pois atingiu 95,4\% da população a ser atendida. É importante notar, que a cobertura mínima na $1^{\mathrm{a}}$ etapa foi no Município de Jutaí, com $74,6 \%$ de cobertura, enquanto para 48 municípios o grau de cobertura foi de $100 \%$.

Já para a $2^{\mathrm{a}}$ dose da campanha, identificou-se a queda de 5,6\% da efetivação da cobertura vacinal em relação à população alvo, sendo beneficiadas 97.584 adolescentes. Em relação ao número total de adolescentes aptas para a vacinação, a cobertura foi de $94,4 \%$. Nesse caso, o número mínimo foi de $75,4 \%$ de

Dados coletados até o dia 11 de julho de 2014. Ressalta-se que a terceira dose de campanha ainda não havia sido encerrada até a finalização do estudo. cobertura no município de São Gabriel da Cachoeira, e com grau de $100 \%$ de cobertura em 19 municípios. Ou seja, a queda foi significativa, mas o alto grau de cobertura nos permite concluir que ela atingiu, nesse ponto, o objetivo social de vacinar essa parcela da população contra o HPV no estado do Amazonas. No entanto, para imunização contra o HPV se faz necessário o encerramento do ciclo das três doses da vacina nas adolescentes para que elas possam ser consideradas imunizadas. Surge, nesse aspecto, o desafio de conseguir manter uma cobertura dos $90 \%$ da campanha de vacinação até o término.

Nessa conjuntura, na terceira dose, constatou-se que 82.065 adolescentes foram beneficiadas, obtendo cobertura de $84,1 \%$ da população alvo imunizadas com a $3^{\text {a }}$ dose. Todavia, a campanha ainda não se encerrou, pois, o objetivo da FVS/SUSAM é finalizar a vacinação até atingir os $100 \%$ de cobertura.

De modo parcial, foi constatado, por meio dos dados, que dos 62 municípios que houve a efetivação da vacina, em 4 deles, na primeira campanha, não foi verificado o critério mínimo de sucesso de $90 \%$ da cobertura vacinal entre adolescentes. Na segunda etapa, 9 dos municípios tiveram dificuldades para a cobertura mínima. E, na terceira etapa, parcialmente, 15 municípios não atingiram a cobertura mínima. Levando em consideração a população a ser vacinada, de 108.283 , destaca-se que $75,78 \%$ receberam o esquema vacinal da $1^{\mathrm{a}} ; 2^{\mathrm{a}}$ e $3^{\mathrm{a}}$ dose e encontram-se totalmente imunizadas.

As coberturas totais de políticas públicas em saúde no interior do estado do Amazonas são permeadas por adversidades ambientais geográficas e climáticas. No que tange o processo de realização da campanha de vacinação contra o HPV, encontram-se dificuldades geográficas para deslocamento da população e recurso financeiro escasso, tudo isso por conta das cheias e vazantes dos rios, características ambientais tipicamente amazônicas. Na primeira etapa, realizada no mês julho, é a vazante dos rios, o que facilita o deslocamento das equipes a todas as escolas onde se encontram as adolescentes. Na segunda etapa, realizada no mês de setembro de 2013, os rios no Estado do Amazonas encontram-se secos, o que para alguns municípios inviabiliza o deslocamento da equipe de saúde para navegar e chegar até as escolas. E, por fim, na terceira etapa, é o período das cheias dos rios, em que também, apresenta dificuldades para o deslocamento das equipes. 
Nesse período, por vezes, há escolas que por estarem submersas nos rios, acabam suspendendo as suas atividades.

\section{Estimativa dos custos diretos da campanha de vacinação para o estado do Amazonas}

A partir dos resultados acerca das adolescentes contempladas, apresenta-se o custo de vacina com a logística no estado do Amazonas, referente às três etapas de efetivação da campanha contra o HPV.

Os dados apontaram que cada vacina teve um valor de comercialização de $\mathrm{R} \$ 42,00$ quando comprada pela instância estadual, a SUSAM, por meio do fabricante GlaxoSmithKline. Constatou-se, deste modo, que para a realização da campanha, foram compradas 324.849 doses para as 3 etapas, que inicialmente, por meio do fabricante, tiveram um custo de $\mathrm{R} \$ 13.643 .658,00$. Todavia, o custo unitário da vacina teve um valor diferenciado para cada município. Pois as condições geográficas de deslocamento para os municípios são diversas e, muitos variam a forma de acesso sendo por via área, terrestre e/ou fluvial.

A tabela 2 apresenta o custo da logística e mais o custo da vacina comprada diretamente no fornecedor para estimar o custo direto da campanha contra o HPV no estado do Amazonas.

Tabela 2 - Custos da campanha de vacinação contra o HPV no Amazonas.

\begin{tabular}{ll}
\hline Quantidade de Vacinas Adquiridas & 324.849 \\
Custo Unitário da Vacina & $\mathrm{R} \$ 42,00$ \\
Total & RS 13.643.658,00 \\
Custo da entrega da vacina & $\mathrm{R} \$ 171.817,79$ \\
$\begin{array}{l}\text { Contrapartida estadual para os } \\
\text { municípios }\end{array}$ & $\mathrm{R} \$ 2.275 .830,00$ \\
$\begin{array}{l}\text { Custo de material de insumos } \\
\text { Total }\end{array}$ & $\mathrm{R} \$ 58.509,00$ \\
\hline TOTAL DOS CUSTOS & $\mathbf{R} \mathbf{2 . 5 0 6 . 1 5 6 , 7 9}$ \\
\hline DIRETOS DA VACINA & $\mathbf{R} \$ \mathbf{1 6 . 1 4 9 . 8 1 4 , 7 9}$ \\
\hline
\end{tabular}

Verificou-se que o estado destinou para os municípios valores de contrapartida com vistas a viabilizar a realização das três etapas da campanha de vacinação. Ressalta-se que a capital assumiu toda a despesa da logística, não havendo contrapartida do Estado.

Diante das especificidades geográficas da Amazônia, o custo unitário da vacina em 36 municípios, ficou entre $\mathrm{R} \$ 50,00$ a $\mathrm{R} \$ 59,00$ reais. Já em 23 desses municípios, a média ficou entre $\mathrm{R} \$ 60,00$ a $\mathrm{R} \$ 64,65$ e abaixo de $\mathrm{R} \$ 50,00$ foram 3 municípios do interior, incluindo a capital. Diante desses achados, afirma-se que a estimativa de custos diretos para o estado do Amazonas, na realização da campanha contra o HPV, teve o valor de R\$16.149.814,79 para o Estado.

A matriz de correlação de Pearson, considerando os custos apresentados na tabela 3 , revelou que a variável população alvo correlacionou-se significativamente com os custos. Neste caso, destaca-se que o aumento do número da população alvo (beneficiárias) está correlacionado com o aumento de custos.

Tabela 3 - Matriz de Correlação de Pearson para os custos das campanhas de vacinação contra o HPV no Amazonas, 2013-2014

\begin{tabular}{|c|c|c|c|c|}
\hline Variáveis & Custos & $\begin{array}{l}\text { Distância } \\
(\mathrm{Km}) \text { em } \\
\text { Linha Reta } \\
\text { da Capital }\end{array}$ & $\begin{array}{c}\text { Área } \\
\text { Territorial } \\
\left(\mathbf{K m}^{2}\right)\end{array}$ & $\begin{array}{l}\text { População } \\
\text { Alvo }\end{array}$ \\
\hline Custos & 1,00 & 0,510 & 0,652 & $<0,001^{\star}$ \\
\hline Distância em & & & & \\
\hline $\begin{array}{l}\text { Linha Reta }(\mathrm{Km}) \\
\text { da Capital }\end{array}$ & 0,510 & 1,00 & 0,612 & 0,432 \\
\hline $\begin{array}{l}\text { Área Territorial } \\
\left(\mathrm{Km}^{2}\right)\end{array}$ & 0,652 & 0,612 & 1,00 & 0,529 \\
\hline População Alvo & $<0,001^{\star}$ & 0,432 & 0,529 & 1,00 \\
\hline
\end{tabular}

*Correlação significante: $\mathrm{p} \leq 0,05$

Ressalta-se ainda que a variável área territorial $\left(\mathrm{km}^{2}\right)$ e custo da vacina apresentaram correlações inversas ainda que não significativas $(-0,108 ; \mathrm{p}=0,612)$. Isto é, evidenciou-se que quanto maior a área territorial $\left(\mathrm{km}^{2}\right)$ do município, menor foi o custo indireto da campanha de vacinação no estado do Amazonas. Bem como, quanto maior à distância em linha reta da capital 
também foi menor o custo da efetivação da campanha, conforme a correlação inversa não significativa encontrada $(-0,156 ; \mathrm{p}=0,510)$.

\section{Discussão}

Acerca da cobertura vacinal contra o HPV das adolescentes, essa política vem sendo discutida no campo da saúde e políticas públicas, de modo que eles tenham acesso gratuito como dispositivo de prevenção do câncer no colo do útero na população. (16) Haja vista que a vacina já é disponível em clínicas particulares com um elevado custo.(17)

No Brasil, no ano de 2006, foi autorizada a comercialização das vacinas contra o HPV, chegando a custar 100 dólares por dose.(18-19) Na Europa, o custo da vacina é de 96 euros e nos Estados Unidos, o custo unitário é de 120 dólares.(19) De acordo com o estudo de Carvalho(20), já existia a disponibilidade gratuita dessas vacinas no ano 2011, mesmo que de modo muito particular de cada cidade. Por exemplo, São Francisco do Conde no Estado da Bahia foi a cidade pioneira e implantou antes de ser introduzido no PNI.

Embora seja uma vacina de alto custo, a adesão da vacinação é permeada por diversos fatores desafiadores como fatores individuais e externos. Referindo-se a fatores individuais, têm-se a aceitabilidade dos pais e dos próprios indivíduos, vinculada a valores, crenças e informações sobre a epidemiologia do HPV, do câncer, da vacina e das possibilidades de acesso. Quanto aos fatores externos se têm a atitude dos profissionais de saúde, fatores organizacionais, comunitários, econômicos e sociais.(17)

A cobertura das vacinas das adolescentes no estado do Amazonas é um dado novo para a literatura, pois ainda há lacuna no estado da arte sobre esse tipo de estudo, haja vista que no Brasil, apenas em 2014 foi disponibilizada no PNI a implantação desta campanha. No cenário internacional, existem países que já tem implantado há anos o programa de vacinação, como é o caso dos Estados Unidos, Panamá, Peru, Holanda e Suíça que atingem $70 \%$ da cobertura da vacinação contra o HPV.(16) Esses dados coadunam com o percentual de $75,78 \%$ de cobertura das vacinas realizadas até a finalização desta pesquisa.

Faz-se necessário destacar os desafios das implantações de políticas de saúde na Amazônia.(21) Merecem destaque algumas peculiaridades da região Amazônica, como, por exemplo, a sazonalidade do período de cheia e vazante dos rios, processos de desmatamentos e queimadas, condições climáticas, entre outros, que interferem diretamente no processo de mobilidade urbana e rural na Amazônia. Essas peculiaridades da Amazônia supracitadas e a escassez de recursos humanos capacitados foram os itens encontrados como fatores desafiadores para os gestores municipais para a realização das campanhas de vacinação e a cobertura esperada de $100 \%$ de meninas imunizadas.

O orçamento das ações de saúde abarca olhares interdisciplinares no contexto amazônico, exigem planejamento interdisciplinar, dentre eles profissionais da saúde, geógrafos, administradores, economistas, etc., pois há necessidade da organização nas formas de planejar e calcular um orçamento para o cumprimento da distribuição equânime entre os municípios de repasse de verbas e, portanto, efetivar o pacto de gestão em saúde.(22-23)

Aponta-se que as vantagens dos estudos de estimativa de custos podem ser utilizadas em desdobramentos de campanhas semelhantes, bem como em outras campanhas cujos desdobramentos sejam diferentes, mas que mesmo assim podem ser comparados.(24)

Destaca-se que na literatura, estudos de avaliação econômica sobre estimativas de custos de vacinas são escassos. O Boletim Brasileiro de Avaliação e Tecnologias em Saúde(25) observa que as revisões sistemáticas, revelam que a vacina contra HPV, quando combinada aos métodos de rastreio tradicionais, é custo-efetiva para controle do câncer de colo uterino. Ainda de acordo com este boletim, foram reveladas informações para estimar o impacto orçamentário para a vacinação contra o HPV no Brasil. Logo é revelado o valor monetário da dose na moeda nacional, estaria orçado em torno de $\mathrm{R} \$ 25,00$, mas a realidade encontrada no Estado do Amazonas foi diferente ao estimado, cujo valor médio foi de R $\$ 58,72$.

Estudos do Ministério da Saúde para implantação da vacinação contra o HPV para o primeiro ano de campanha foi estimado em 160 milhões de reais, que comprometeria $25 \%$ do orçamento total do PNI. (25) Neste contexto, os custos diretos do estado do Amazonas, comparando com o valor estimado, comprometeu $10 \%$ do orçamento planejado.

Os custos de vacinação para prevenção apresentamse elevados.(17,25-26) No entanto, o que está em questão é a prevenção do câncer, pois o custo com a prevenção do HPV, por meio da vacinação, se constitui 
um valor menor do que o tratamento para mulheres acometidas pelo câncer no colo do útero.(15,27-28)

Estudos, neste aspecto, têm abordado gastos em saúde relacionados ao câncer de colo uterino para subsidiar análises de custo-efetividade de programas de vacinação. Nesta acepção Goldie et al.(29); Ben Gobrane et al.(30) e Fonseca et al.(7), evidenciaram que o custo anual por paciente com câncer de colo uterino foi de R \$ 8.711,00 (aproximadamente US\$ $4.970,00)$, compatível com o custo estimado por caso de câncer de colo uterino no Brasil em estudo de custo-efetividade para vacinação contra o HPV (US\$ 4.500,00).

Por fim, discute-se, a partir da hipótese confirmada, que quanto maior o número de adolescentes vacinadas, aumenta-se o tempo para planejamento e realização da campanha, bem como, o quantitativo de pessoas envolvidas na efetivação, logo irão interferir nos custos. Sendo assim, essa evidência coaduna com a ampliação financeira executada pelo Ministério da Saúde ao investir mais R \$360,7 milhões na aquisição de 12 milhões de doses, por ampliar a faixa etária de meninas a serem vacinadas.(16)

\section{Considerações Finais}

Os resultados obtidos podem ser considerados como satisfatórios ao contemplar um conhecimento sobre o custo da efetivação de uma campanha de vacinação, por considerar que se chegou a um resultado final e pôde-se fazer um conjunto de análise econômica e social em saúde. E com isso, trazer um produto, na qual os gestores em saúde possam se basear para planejar ações semelhantes e inovadoras a partir desses resultados.

Desta maneira, pode-se afirmar que este estudo traz contribuições no campo da economia em saúde, para fins de mensuração e planejamento estratégico do custo de implantação de futuras políticas públicas de saúde, sobretudo, porque serve como um subsídio para gestores em saúde que estão em processo de implementação do Programa Nacional de Imunização (PNI) para a vacina do HPV. Este estudo traz também como contribuição, informações e conhecimentos que auxiliem a tomada de decisão e uma ampliação planejada da oferta de serviços e produtos vinculados à gestão em saúde.

A Economia da Saúde, neste aspecto, se insere neste contexto como um campo de produção de conhecimento interdisciplinar que tem ajudado a promover a análise da eficiência de inovações tecnológicas e gestão em saúde, buscando alternativas que aliem o atendimento das necessidades terapêuticas à disponibilidade de recursos financeiros. E, com isso, as verbas destinadas à campanha de promoção e prevenção de saúde pública, possam cumprir por meio do custo benefício o papel social de prevenção e promoção de saúde e atingir maior cobertura vacinal.

Quanto à maior cobertura de campanhas de vacinação, a escola pode ser uma grande aliada nessas questões educacionais, de conscientização, saúde e de transformações sociais, pois ela ainda é uma das instituições que mais interferem no pensamento e no agir dos cidadãos. Portanto, o agir-educativo-cuidativo pode ser uma forma de potencializar ações de saúde e promover ações exitosas envolvendo a comunidade escolar e a família na prevenção, esse trabalho feito em conjunto com a instituição de ensino e a sociedade certamente fará um efeito mais eficaz.

O gestor em saúde, no planejamento de produção e/ou efetivação de tecnologias e inovações em saúde, deve levar em consideração a complexidade e dinamicidade tanto dos territórios quanto dos seres humanos, bem como, propor à prática profissional perspectivas mais implicadas com a realidade e com os processos coletivos. Este estudo revelou que vacinar no contexto amazônico, torna-se muito oneroso. Pois, tomando-se como base o valor estimado para ser gasto no primeiro ano de campanha pelo Ministério da Saúde, fere o princípio de equidade ${ }^{5}$ no planejamento das ações de saúde.

Conclui-se, nesta perspectiva, que este trabalho contribuiu para um olhar da prática organizativa dos gestores, principalmente, na implantação de tecnologias e inovações em saúde. Haja vista que o estado do Amazonas antecipou o calendário de campanha, pois no ano de 2013, ano das duas primeiras etapas de campanha, ainda não havia sido aprovado a efetivação pelo calendário nacional de vacinação, por meio do PNI, a campanha nacional do HPV.

\footnotetext{
5"Apesar de existir um problema terminológico na variedade conceitual de equidade e, em alguns casos, problemas também com o significado da expressão quando utilizado no sentido das desigualdades, percebe-se, de modo geral, que há consenso ou aceitação ampla na literatura da definição formulada por Whitehead, ou seja, equidade em saúde, para a autora, remete à noção de que, de acordo com os ideais, todos os indivíduos de uma sociedade devem ter justa oportunidade para desenvolver seu pleno potencial de saúde e, no aspecto prático, ninguém deve estar em desvantagem para alcançá-lo. Consequentemente, equidade em saúde refere-se à redução das diferenças consideradas desnecessárias, evitáveis, além de serem consideradas injustas." (31:59)
} 


\section{Referências}

1 Lima GR, Girão MJBC, Baracat EC. Papilomavírus humano e o câncer de colo uterino. In: Ginecologia de Consultório. 1. ed. São Paulo: Editora de Projetos Médicos; 2003. p. 213-218.

2 Linhares AC, Villa LL. Vacinas contra Rotavírus e Papiloma vírus humano (HPV). J Pediatr. 2006 jul;82(3):25-34. doi: 10.1590/S002175572006000400004

3 Fonseca AJ. Ensaios sobre a economia do câncer do colo do útero: teorias e evidências para o caso de Roraima [dissertação]. Porto Alegre: Universidade Federal do Rio Grande do Sul; 2011.

4 Diretoria de Vigilância Epidemiológica (BR). Programa Estadual de Imunizações- SC. Informe técnico sobre a vacina papilomavírus humano (HPV) na atenção básica. Santa Catarina: DIVE; 2014.

5 Instituto Nacional de Câncer José Alencar Gomes da Silva. Coordenação Geral de Ações Estratégicas. Estimativa 2016-2017: incidência de câncer no Brasil. Rio de Janeiro: INCA; 2017.

6 Instituto Nacional de Câncer José Alencar Gomes da Silva. Estimativa 2014: incidência de câncer no Brasil. Rio de Janeiro: INCA; 2014.

7 Fonseca AJ, Ferreira LP, Dalla-Benetta AC, Roldan, CN, Ferreira MLS. Epidemiologia e impacto econômico do câncer de colo de útero no Estado de Roraima: a perspectiva do SUS. Rev. Bras. Ginecol. Obstet. 2010;32(8):386-92. doi: http:// dx.doi.org/10.1590/S0100-72032010000800005

8 Zahdi MR, Maluf Junior I, Maluf EMCP. Hepatite A: avaliação do custo-benefício da prevenção pela vacina - Paraná, Brasil. Rev Bras Med Fam Comunidade. 2010;4(16):281-8. doi: https://doi. org/10.5712/rbmfc4(16)420

9 Valentim J, Sartori AM, Soárez PC, Amaku M, Azevedo RS, Novaes HM. Cost-effectiveness analysis of universal childhood vaccination against varicella in Brazil. Vaccine. 2008 Nov;26(49):628191. doi: 10.1016/j.vaccine.2008.07.021
10 Itria A, Novaes HMD, Soárez PC, Nobrega LAL, Sartori AMC. A importância dos métodos de custeio e valoração nas avaliações econômicas em saúde: repercussões sobre os resultados de avaliação da vacina antimeningocócica C. Physis. 2012 abr-jun;22(2):641-58. doi: 10.1590/S010373312012000200013

11 Ryman K, Dietz V, Cairns L. Too little but not too late: results of a literature review to improve routine immunization programs in developing countries. BMC Health Serv Res. 2008 Jun;8:134. doi: $10.1186 / 1472-6963-8-134$

12 Wallace A, Dietz V, Cairns L. Integration of immunization services with other health interventions in the developing world: what works and why? Systematic literature review. Trop Med Int Health. 2009 Jan;14(1):11-19. doi: 10.1111/j.1365-3156.2008.02196.x

13 Vanni T, Luz PM, Ribeiro RA, Novaes HMD, Polanczyk CA. Avaliação econômica em saúde: aplicações em doenças infecciosas. Cad. Saúde Pública. 2009 dez;25(12):2543-52. doi: 10.1590/ S0102-311X2009001200002

14 Polit DF, Beck CT. Fundamentos de pesquisa em enfermagem: avaliação de evidências para a prática da enfermagem. Porto Alegre: Artmed; 2011.

15 Wichmann RB, Silva MT. Impacto orçamentário da introdução da vacina HPV no SUS: uma ferramenta para formuladores de políticas. J. Bras. Econ. Saúde. 2012;4(3):424-329.

16 Ministério da Saúde (BR). Vacina contra HPV na prevenção de câncer de colo de útero. Brasília; 2013

17 Novaes HMD. A vacina contra HPV e o câncer de colo de útero: desafios para a sua incorporação em sistemas de saúde. Rev. bras. epidemiol, 2008 set;11(3):524-5. doi: 10.1590/S1415$790 \times 2008000300022$

18 Bragagnolo AL, Eli D, Haas P. Papiloma Vírus Humano (HPV). Rev. Bras. Anal. Clinica. 2010;42(2):91-6. 
19 Borsatto AZ, Vidal MLB, Rocha RCNP. Vacina contra o HPV e a Prevenção do Câncer do Colo do Útero: Subsídios para a Prática. Rev Bras Cancerol. 2011;57(1):67-74.

20 Carvalho IS. Custo-utilidade da vacinação contra Papilomavírus Humano no Brasil. [dissertação]. Salvador: Universidade Federal da Bahia; 2013.

21 Viana ALÁ, Machado CV, Baptista TWF, Lima LD, Mendonça MHM, Heimann LS et al. Sistema de saúde universal e território: desafios de uma política regional para a Amazônia Legal. Cad. Saúde Pública. 2007;23(2):117-31. doi: 10.1590/ S0102-311X2007001400002

22 Ministério da Saúde (BR). Política nacional de atenção integral à saúde da mulher: princípios e diretrizes [Internet]. Brasília: Ministério da Saúde; 2004 [citado 2014 abr 12]. Disponível em: http:// conselho.saude.gov.br/ultimas_noticias/2007/ politica_mulher.pdf

23 Ministério da Saúde (BR). Sistema de Planejamento do SUS: uma construção coletiva. Plano Nacional de Saúde (PNS) 2008/2009-2011. Brasília: Ministério da Saúde; 2010

24 Rascati KL. Introdução à farmacoeconomia. Porto Alegre: Artemed; 2010

25 Boletim Brasileiro de Avaliação de Tecnologias em Saúde. Câncer de colo de útero: a vacina para prevenção do HPV e o desafio para a melhoria da qualidade do rastreamento no Brasil [internet]. BRATS. 2011 [citado 2014 jul 5];6(17):1-14. Disponível em: http://portal.anvisa.gov.br/wps/ $\mathrm{wcm} /$ connect/86bc4b8044ae52a99609b66b0d9f1 $4 \mathrm{~d} 3 / \mathrm{C} \% \mathrm{C} 3 \% 82 \mathrm{NCER}+\mathrm{DE}+\mathrm{COLO}+\mathrm{DE}+\% \mathrm{C} 3 \% 9 \mathrm{~A}$ TERO+Sem+logo.pdf?MOD=AJPERES

26 Fonseca AJ, Ferreira LP, Dalla-Benetta AC, Roldan $\mathrm{CN}$, Ferreira MLS. Epidemiologia e impacto econômico do câncer de colo de útero no Estado de Roraima: a perspectiva do SUS. Rev. Bras. Ginecol. Obstet. 2010;32(8):386-392. doi: 10.1590/S010072032010000800005

27 Pinto AP, Túlio S, Cruz OR. Co-fatores do HPV na oncogênese cervical. Rev. Assoc. Med. Bras. 2002;48(1):73-8.
28 Fonseca AJ, Ferreira LCL, Balbinotto Neto G. Cost-effectiveness of the vaccine against human papillomavirus in the brazilian Amazon region. Rev. Assoc. Med. Bras. 2013;59(5):442-451. doi: 10.1016/j.ramb.2013.03.004

29 Ben Gobrane HH, Aounallah-Skhiri H, Oueslati F, Frikha H, Achour N, Hsairi M. Estimated cost of managing invasive cervical cancer in Tunisia. Sante Publique. 2009 Nov-Dec;21(6):561-9.

30 Viana AL, Fausto MCR, Lima LD. Política de saúde e equidade. São Paulo Perspec.2003 jan-mar;17(1):58-68. doi: 10.1590/S010288392003000100007
Recebido em: 3 ago. 2017

Aceito em: 20 nov. 2017 
Magalhães, P. da S.; et al. 